\title{
Cluster Analysis and Finite-Size Scaling for Ising Spin Systems
}

\author{
Yusuke Tomita and Yutaka Okabe* \\ Department of Physics, Tokyo Metropolitan University, Hachioji, Tokyo 192-0397, Japan \\ Chin-Kun $\mathrm{Hu}+$ \\ Institute of Physics, Academia Sinica, Nankang, Taipei 11529, Taiwan
}

\begin{abstract}
Based on the connection between the Ising model and a correlated percolation model, we calculate the distribution function for the fraction $(c)$ of lattice sites in percolating clusters in subgraphs with $n$ percolating clusters, $f_{n}(c)$, and the distribution function for magnetization $(m)$ in subgraphs with $n$ percolating clusters, $p_{n}(m)$. We find that $f_{n}(c)$ and $p_{n}(m)$ have very good finite-size scaling behavior and they have universal finite-size scaling functions for the model on square, plane triangular, and honeycomb lattices when aspect ratios of these lattices have the proportions $1: \sqrt{3} / 2: \sqrt{3}$. The complex structure of the magnetization distribution function $p(m)$ for the system with large aspect ratio could be understood from the independent orientations of two or more percolation clusters in such system.
\end{abstract}

PACS numbers: 05.50.+q, 64.60.Ak, 75.10.-b

Universality and scaling are two important concepts in the modern era of critical phenomena [1] and for analyzing the simulation or experimental data of finite critical systems, one often appeals to finite-size scaling [2 [4] where both critical exponents and finite-size scaling function play important role. The universality of critical exponents was well known for a long time [1] , but the universality of finite-size scaling functions received much attention only in recent years 5 [10]. In 1984, Privman and Fisher first proposed the idea of universal finite-size scaling functions (UFSSF's) with nonuniversal metric factors [3]. In 1995 1996, Hu, Lin and Chen (HLC) 50 applied a histogram Monte Carlo simulation method [11] to calculate the existence probability [11] (also called crossing probability [12) $E_{p}$, the percolation probability $P$, the probability for the appearance of $n$ percolating clusters $W_{n}$ [5] of site and bond percolation on finite square (sq), plane triangular (pt), and honeycomb (hc) lattices, whose aspect ratios approximately have the relative proportions $1: \sqrt{3} / 2: \sqrt{3}$ considered by Langlands, et al. [12]. Using nonuniversal metric factors, HLC found that the six percolation models have very nice UFSSF's for $E_{p}, P$, and $W_{n}$ near the critical points [5], and at the critical point the average number of percolating clusters increases linearly with aspect ratios of the lattices [5]. Using Monte Carlo simulation, Okabe and Kikuchi found UFSSF's for the Binder parameter $g$ [13] and magnetization distribution functions $p(m)$ of the Ising model on planar lattices [6], and Wang and $\mathrm{Hu}$ found UFSSF's for dynamic critical phenomena of the Ising model [7]. Based on the connection between the $q$-state bond correlated percolation model (BCPM) and the $q$-state Potts model [14] Hu, Chen, and Lin found UFSSF's for $E_{p}$ and $W_{n}$ of the $q$ state BCPM without using nonuniversal metric factors [9]. It is of interest to get a deeper understanding of the UFSSF's for $W_{n}$ and $P$ for the system with multiple percolating clusters.

In this paper, based on the connection between the Ising model, i.e. the two-state Potts model, and the twostate BCPM, we use Monte Carlo method to calculate the distribution function for the fraction $(c)$ of lattice sites in percolating clusters in subgraphs with $n$ percolating clusters, $f_{n}(c)$, and the distribution function for magnetization $(m)$ in subgraphs with $n$ percolating clusters, $p_{n}(m)$. We find that $f_{n}(c)$ and $p_{n}(m)$ have very good finite-size scaling behavior and they have UFSSF's for the model on sq, pt, hc lattices when aspect ratios of these lattices have the proportions $1: \sqrt{3} / 2: \sqrt{3}$. Since $W_{n}$ and $P$ of the two-state BCPM for the Ising model may be calculated from $f_{n}(c)$, the universality of finite-size scaling functions for $W_{n}$ and $P$ are related to universality of finite-size scaling functions for $f_{n}(c)$. The complex structure of $p(m)$ for the system with large aspect ratio could be understood from the independent orientations of two or more percolation clusters in such system. Our work suggests many problems for further research.

The Hamiltonian of the Ising model on an $L_{1} \times L_{2}$ lattice $G$ of $N_{b}$ bonds is given by $\mathcal{H}=-J \sum_{<i, j>} \sigma_{i} \sigma_{j}$ $h \sum_{i} \sigma_{i}$, where $\sigma_{i}= \pm 1, J>0$ and is the ferromagnetic coupling constant between the nearest-neighbor Ising spins, and $h$ is the external magnetic field. Using subgraph expansion, $\mathrm{Hu}$ [14] showed that the partition function of the Ising model on $G$ may be written as

$$
\begin{aligned}
Z_{N}=e^{K N_{b}} & \sum_{G^{\prime} \subseteq G} p^{b\left(G^{\prime}\right)}(1-p)^{N_{b}-b\left(G^{\prime}\right)} \\
& \times \prod_{\text {cluster }}^{\prod^{\prime}}\left[2 \cosh \left(B n_{c}\left(G^{\prime}\right)\right)\right],
\end{aligned}
$$

where $p=1-e^{-2 K}, K=J /\left(k_{B} T\right), B=h /\left(k_{B} T\right)$, $b\left(G^{\prime}\right)$ is the number of occupied bonds in $G^{\prime}$, and the sum is over all subgraphs $G^{\prime}$ of $G$, the product extends over all clusters in a given $G^{\prime}, n_{c}\left(G^{\prime}\right)$ is the number of sites in each cluster. When $B=0$, Eq. (1D) reduces to the result of Ref. [15]. The sites connected by occupied bonds are in the same cluster; all spins in a cluster must be in the same direction, which may be up or 
down. Using $Z_{N}$ of Eq. (11), Hu found that the spontaneous magnetization and the magnetic susceptibility of the Ising model are related to the percolation probability $P$ and the mean cluster size of the (two-state) BCPM such that the probability weight for the appearance of a subgraph $G^{\prime}$ of $b\left(G^{\prime}\right)$ bonds and $n\left(G^{\prime}\right)$ clusters is given by $\pi\left(G^{\prime}, p\right)=p^{b\left(G^{\prime}\right)}(1-p)^{N_{b}-b\left(G^{\prime}\right)} 2^{n\left(G^{\prime}\right)}$. Such connection ensures that the phase transition of the Ising model is the percolation transition of the BCPM. The extension of the probability weight $\pi\left(G^{\prime}, p\right)$ to a $q$-state Potts model is simply achieved by replacing 2 by $q$ in $\pi\left(G^{\prime}, p\right)$. From $\pi\left(G^{\prime}, p\right)$ we can define

$$
E_{p}(G, p)=\sum_{G_{p}^{\prime} \subseteq G} \pi\left(G_{p}^{\prime}, p\right) / \sum_{G^{\prime} \subseteq G} \pi\left(G^{\prime}, p\right),
$$

which is called the existence probability of the BCPM. Here the sum in the denominator is over all subgraphs $G^{\prime}$ of $G$ and the sum in the numerator is restricted to all percolating subgraphs $G_{p}^{\prime}$ of $G$. In [16], Hu and Chen found that $E_{p}(G, p)$ has very good finite-size scaling behavior. In [9], $\mathrm{Hu}, \mathrm{Chen}$, and Lin found that $E_{p}(G, p)$ has UFSSF.

In the present paper we use the Wolff algorithm 17] for spin update and study the percolating properties of clusters on planar lattices with periodic boundary conditions for both directions. For the assignment of a bondpercolating cluster, we consider free and periodic boundary conditions in the vertical and horizontal directions, respectively; that is, a cluster which extends from the top row to the bottom row is a percolating cluster.

We first consider the fraction of lattice sites in the percolating clusters, $c$, and denote the probability distribution function of $c$ by $f(c)$. The average value of $c$ gives the percolation probability $P$,

$$
\langle c\rangle=\int_{0}^{1} c f(c) d c=P,
$$

and plays a role of order parameter in the percolation problem. To study $c$ in subgraphs with exactly $n$ percolating clusters, we decompose $f(c)$ by the number of percolating clusters, $n$; that is,

$$
f(c)=\sum_{n=1}^{\infty} f_{n}(c) .
$$

We should note that

$$
\int_{0}^{1} f_{n}(c) d c=W_{n}, \quad(n=1, \cdots, \infty)
$$

and

$$
\sum_{n=1}^{\infty} W_{n}=1-W_{0}=E_{p}(G, p) .
$$

We may consider the quantity,

$$
\langle c\rangle_{n}=\int_{0}^{1} c f_{n}(c) d c, \quad(n=1, \cdots, \infty)
$$

which is the fraction of lattice sites in the $n$ percolating clusters. In other words, we decompose $\langle c\rangle$ by the number of percolating clusters such that

$$
\langle c\rangle=\sum_{n=1}^{\infty}\langle c\rangle_{n}=P .
$$

The probability distribution of the magnetization, $p(m)$, is an important quantity in the phase transition problem [6.10]. Let us decompose $p(m)$ by the number of percolating clusters in the same way as in $f(c)$,

$$
p(m)=\sum_{n=0}^{\infty} p_{n}(m) .
$$

Now we have the relation,

$$
\int_{-1}^{1} p_{n}(m) d m=W_{n}, \quad(n=0, \cdots, \infty) .
$$

It should be noted that the relation between $f_{n}(c)$ and $p_{n}(m)$ is not a simple one especially for a system with multiple percolating clusters. There are two type of clusters, that is, the cluster with up spins and that with down spins. We may divide the fraction of lattice sites in percolating clusters, $c$, into two classes, $c_{+}$and $c_{-}$. By definition, $c=c_{+}+c_{-}$and to the leading order, $m \sim c_{+}-c_{-}$. If there is only a single percolating cluster, $m \sim c_{+}$or $m \sim c_{-}$; thus, $m^{2} \sim c^{2}$ in the leading contribution. However, if there are two or more percolating clusters, the relation is not trivial, and this is the origin of the complex structure of $p(m)$ for the lattices with large aspect ratio $a$. Therefore, the study of $c$ and $m$ becomes more interesting in the case of multiple percolating clusters.

According to the theory of finite-size scaling [2], if a quantity $Q$ has a singularity of the form $Q(t) \sim t^{\omega}$ near the criticality $t=0$, then the corresponding quantity $Q(L, t)$ for the finite system with the linear size $L$ has a scaling form $Q(L, t) \sim L^{-\omega / \nu} X\left(t L^{1 / \nu}\right)$, where $\nu$ is the correlation-length exponent and is 1 for twodimensional (2D) Ising model. The finite-size scaling is also applicable to the distribution function of $Q$. At the criticality $t=0$, we have a finite-size scaling form $p(Q ; L, t=0) \sim L^{\omega / \nu} Y\left(Q L^{\omega / \nu}\right)$. Thus, we expect the following finite-size scaling relations: $W_{n}(t) \sim$ $X_{n}^{a}\left(t L^{1 / \nu}\right),\langle c\rangle_{n}(t) \sim L^{-\beta / \nu} X_{n}^{b}\left(t L^{1 / \nu}\right), f_{n}(c ; t=0) \sim$ $L^{\beta / \nu} Y_{n}^{a}\left(c L^{\beta / \nu}\right), p_{n}(m ; t=0) \sim L^{\beta / \nu} Y_{n}^{b}\left(m L^{\beta / \nu}\right)$, where $\beta$ is the order-parameter exponent and is $1 / 8$ for $2 \mathrm{D}$ Ising model.

The finite-size scaling functions usually depend on the lattice, or other details of the system. However, with appropriate choices of nonuniversal metric factors $D_{1}$ and $D_{2}$, 


$$
\begin{aligned}
D_{2} Q(L, t) & =L^{-\omega / \nu} \hat{X}\left(D_{1} t L^{1 / \nu}\right), \\
p(Q ; L, t=0) & =D_{2} L^{\omega / \nu} \hat{Y}\left(D_{2} Q L^{\omega / \nu}\right),
\end{aligned}
$$

the finite-size scaling functions $\hat{X}, \hat{Y}$ could become universal. This concept of the UFSSF was first proposed by Privman and Fisher [3], and has been recently confirmed for the percolation problem [5,8] and for the Ising model [6.9. We should note that the UFSSF's still depend on boundary conditions [5].

To study the finite-size scaling and the universality of $f_{n}(c), f(c), p_{n}(m)$, and $p(m)$, we calculate $f_{n}(c), f(c)$, $p_{n}(m), p(m), W_{n},\langle c\rangle_{n},\langle c\rangle, g_{c n}$, and $g_{c}$ for the BCPM on sq, pt, and hc lattices whose aspect ratios approximately have the proportions $1: \sqrt{3} / 2: \sqrt{3}$ and each kind of lattices has two linear dimensions; here $g_{c n}=\langle c\rangle_{n}{ }^{2} /\left\langle c^{2}\right\rangle_{n}, g_{c}=$ $\langle c\rangle^{2} /\left\langle c^{2}\right\rangle$, and the second moments of $c$ are defined as in Eqs. (7) and (3). We note that $g_{c n}$ and $g_{c}$ have the same finite-size scaling property as the Binder parameter 113 .
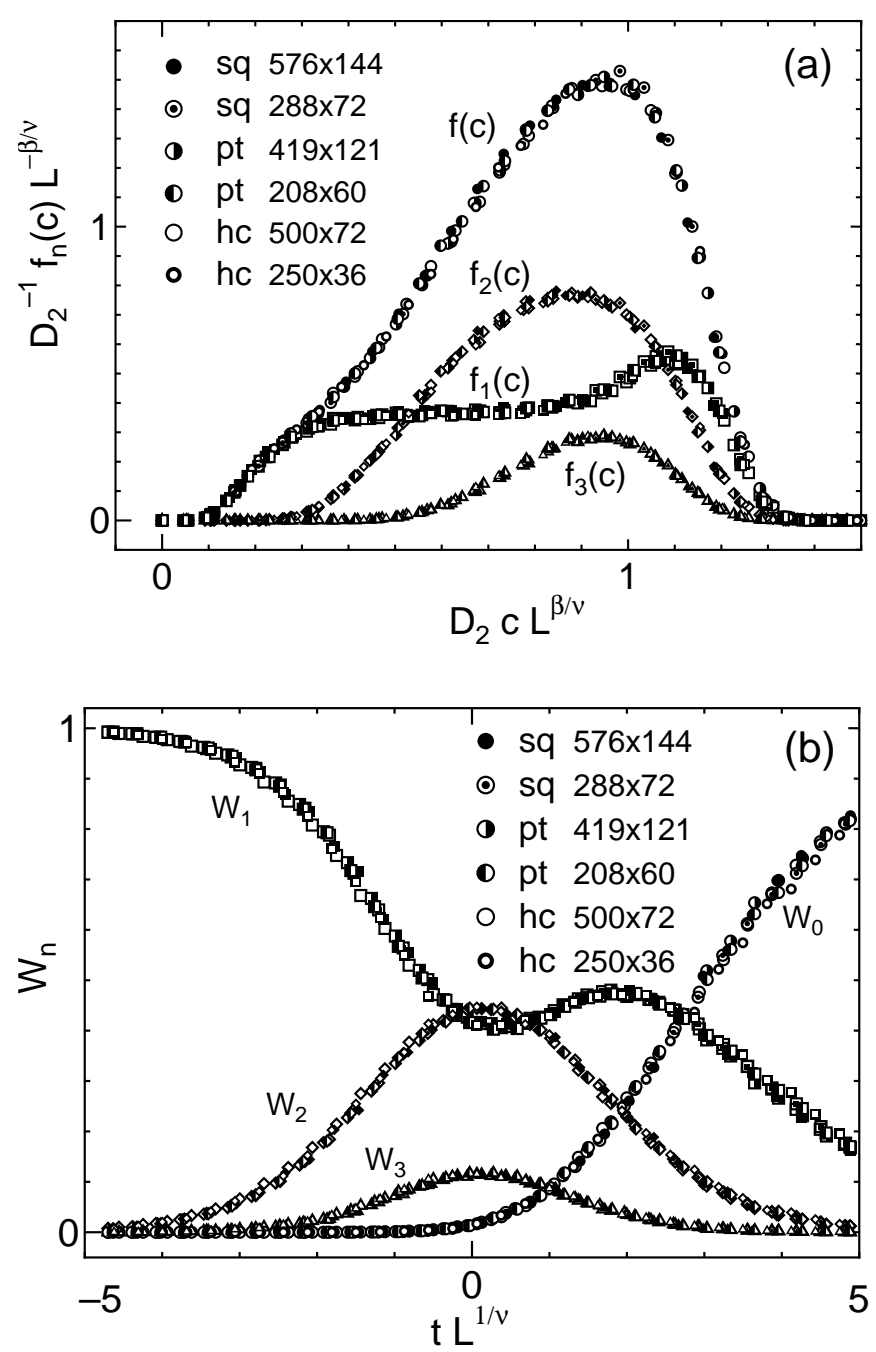
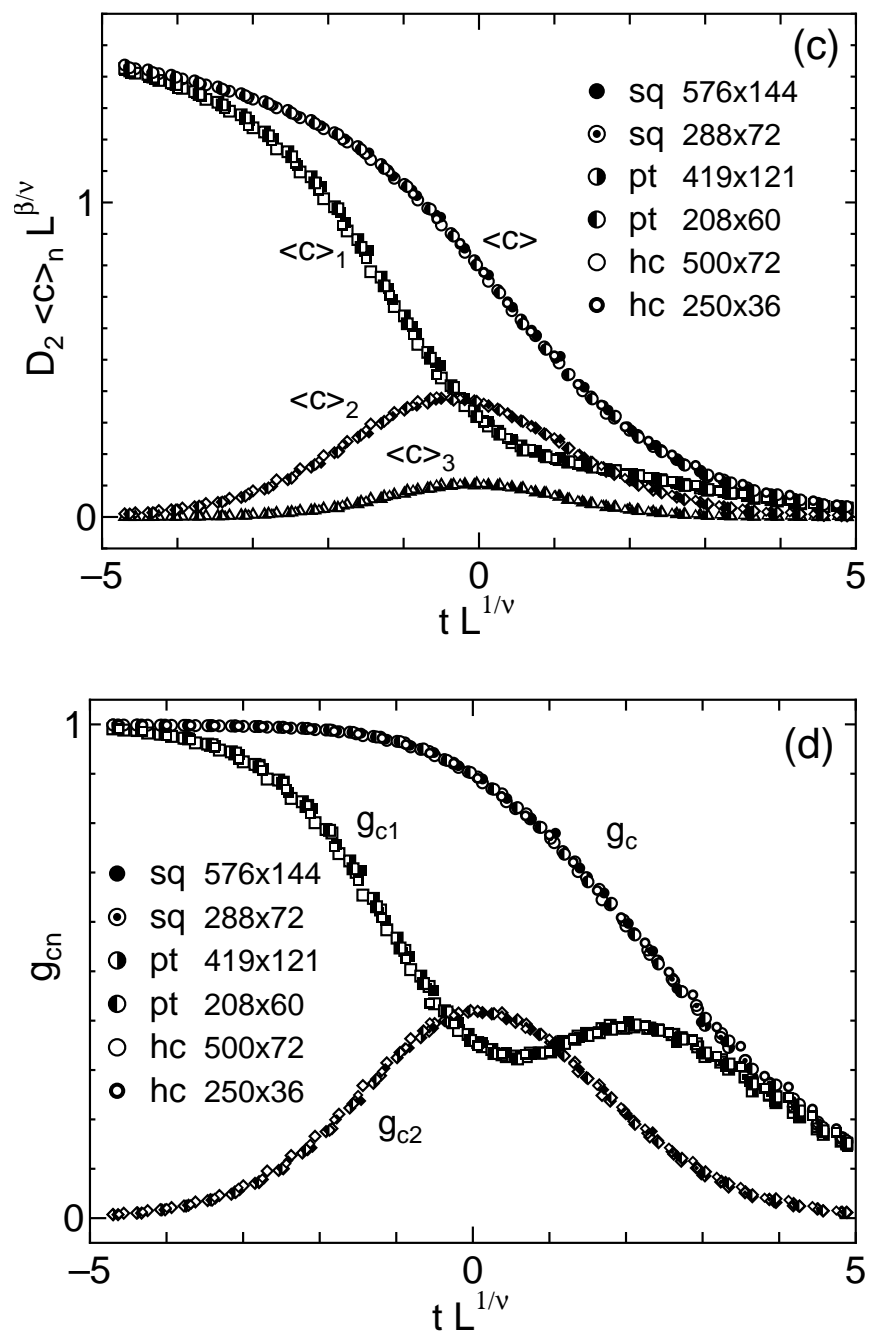

FIG. 1. Universal finite-size scaling functions for the Ising clusters on the sq, pt, and hc lattices. (a) $D_{2}^{-1} f_{n}(c) L^{-\beta / \nu}$ and $D_{2}^{-1} f(c) L^{-\beta / \nu}$ at the critical point as a function of $D_{2} c L^{\beta / \nu}$, (b) $W_{n}$ as a function of $t L^{1 / \nu}$, (c) $D_{2}\langle c\rangle_{n} L^{\beta / \nu}$ and $D_{2}\langle c\rangle L^{\beta / \nu}$ as a function of $t L^{1 / \nu}$, (d) $g_{c n}\left(=\langle c\rangle_{n}^{2} /\left\langle c^{2}\right\rangle_{n}\right)$ and $g_{c}\left(=\langle c\rangle^{2} /\left\langle c^{2}\right\rangle\right)$ as a function of $t L^{1 / \nu}$.

The calculated $D_{2}^{-1} f_{n}(c) L^{-\beta / \nu}$ and $D_{2}^{-1} f(c) L^{-\beta / \nu}$ at the critical point as a function of $D_{2} c L^{\beta / \nu}$ are shown in Fig. 1(a). The lattice sizes are given within the figure. The aspect ratio is $a=4$ for sq lattice, and corresponding equivalent ratios for other lattices. The calculated $W_{n}, D_{2}\langle c\rangle_{n} L^{\beta / \nu}$ (also $D_{2}\langle c\rangle L^{\beta / \nu}$ ), and $g_{c n}$ (also $g_{c}$ ) as a function of $t L^{1 / \nu}\left(t=\left(T-T_{c}\right) / T_{c}\right)$ are presented in Fig. 1 (b), 1 (c), and 11(d), respectively. The calculated $D_{2}^{-1} p_{n}(m) L^{-\beta / \nu}$ as a function of $D_{2} m L^{\beta / \nu}$ is shown in Fig. 2(a). The metric factors $D_{1}$ and $D_{2}$ for the sq lattice are chosen as 1 18. The values of $D_{1}$ for the pt and hc lattices are $1.00 \pm 0.01$ which are consistent with the results of [9]; the values of $D_{2}$ for the pt and hc lattices are $1.02 \pm 0.01$ and $0.98 \pm 0.01$, respectively. Since we have estimated $D_{1}$ as $1.00 \pm 0.01$ for the pt and hc lattices, we have omitted $D_{1}$ in the horizontal axes of the figures. Figures 1 (a)-1 (d) and Fig. 2(a) show that the 
calculated quantities have very good finite-size scaling behavior and the universality is also well satisfied. We should note that the metric factors $D_{1}$ and $D_{2}$ are the same for all quantities.
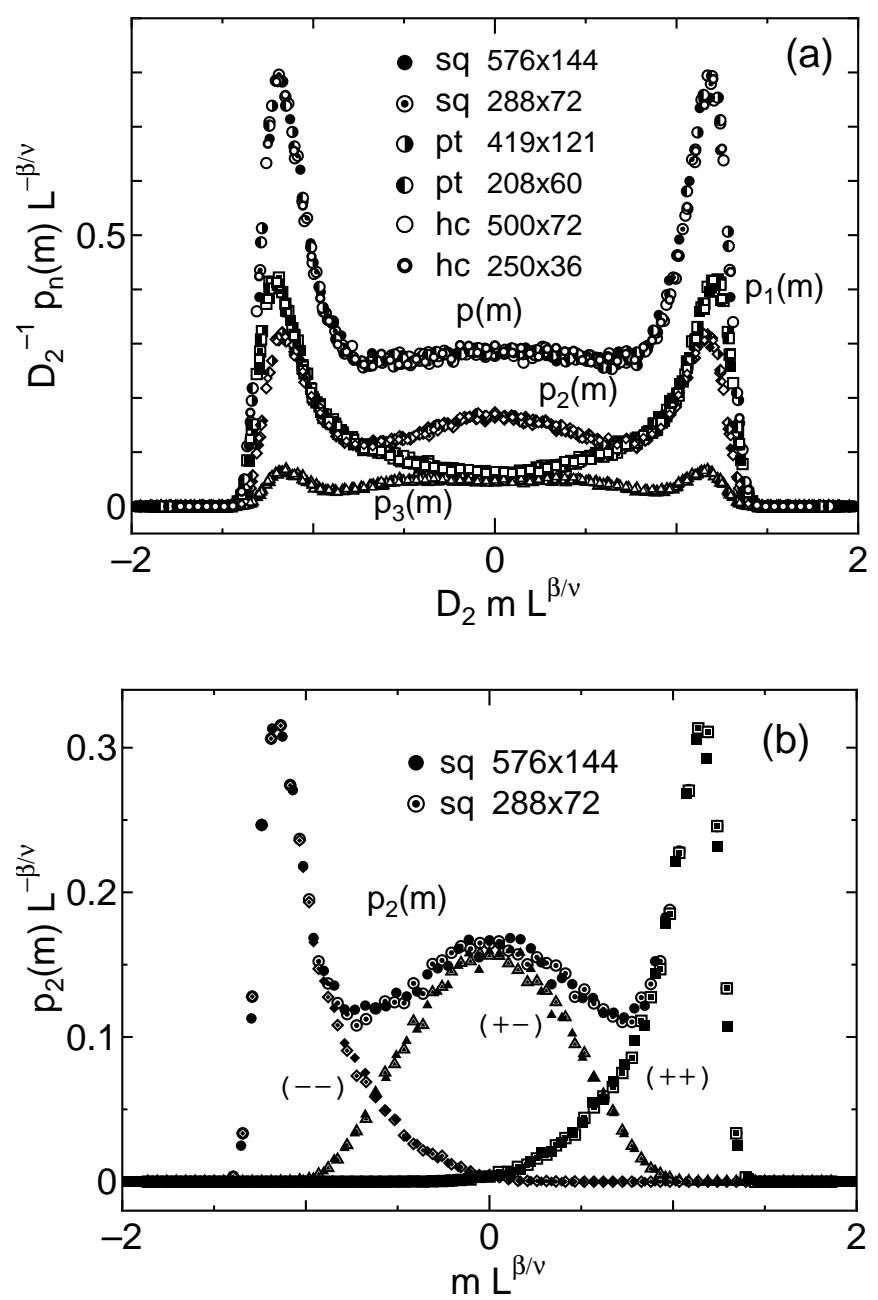

FIG. 2. (a) $D_{2}^{-1} p_{n}(m) L^{-\beta / \nu}$ at $T=T_{c}$ as a function of $D_{2} m L^{\beta / \nu}$. (b) $p_{2}(m)$ at $T=T_{c}$ is decomposed into three classes, $p_{++}(m), p_{--}(m)$ and $p_{+-}(m)$.

Figure 2(a) shows that $p(m)$ at $T=T_{c}$ has a broad peak centered at $m=0$ in addition to two peaks of positive and negative $m$ for the system with the aspect ratio $a=4$ for sq lattice. This is contrast to the case of $a=1$ where $p(m)$ has only two distinct peaks of positive and negative $m$. Such dependence of $p(m)$ on $a$ has already been pointed out in Ref. [10]. From Fig. 2(a), we see that the broad peak of $p(m)$ centered at $m=0$ mainly comes from $p_{2}(m)$. There are two types of Ising clusters, that is, the clusters with up spins or the clusters with down spins. Therefore, if there are many percolating clusters, the combination of the percolating clusters with up spins and those with down spins makes it possible that the total magnetization becomes close to 0 . It is known that the normalized fourth moment of $m$, or the
Binder parameter, at the critical point depends on the aspect ratio 10,19. The origin of such a dependence can be attributed to the structure of many percolating clusters. To clarify this situation, we decompose $p_{2}(m)$ at $T=T_{c}$ into three classes, $p_{++}(m), p_{--}(m)$ and $p_{+-}(m)$ shown in Fig. 2(b). We assign three peaks in $p_{2}(m)$ by the contribution from $p_{++}(m), p_{--}(m)$ and $p_{+-}(m)$. Examples of snapshots of the Ising system with two percolating clusters are presented in Figs. 3(a) and 3(b). In Fig. 3 (a) both percolating clusters are up; in Fig. 3(b) one percolation cluster is up and another percolating cluster is down.

(a)

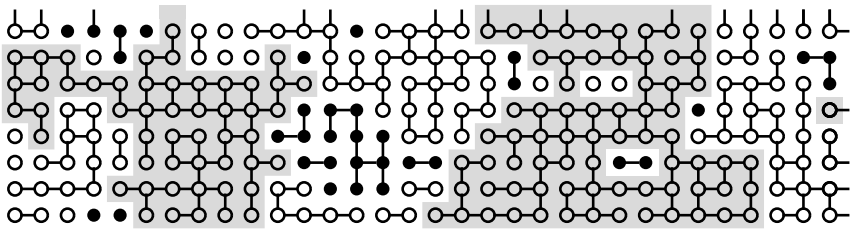

(b)

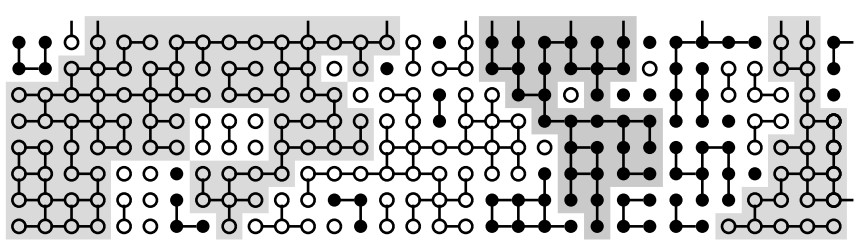

FIG. 3. Examples of snapshots of the Ising system with the aspect ratio $a=4$. Up and down spins are represented by open and closed circles, and active bonds are represented by solid line. Percolating clusters are distinguished by shaded area. There are two percolating clusters with up spins in (a), whereas one percolating cluster is up and the other is down in (b).

From $W_{n}$, we may calculate the average number of percolating cluster by $\langle n\rangle=\sum_{n} n W_{n}$. At the critical point, the values of $W_{n}$ and $\langle n\rangle$ as a function of the aspect ratio $a=L_{1} / L_{2}$ are plotted in Fig. 1 (a) and $⿴$ (b), respectively. We see that $\langle n\rangle$ increases linearly with $a$ for large $a$, which is similar to the case of random percolation [5,20. The slope of $\langle n\rangle$ versus $a$ in Fig. $1(\mathrm{~b})$ is approximately 0.5 .

Following the study of $W_{n}$ for random percolation by $\mathrm{Hu}$ and Lin [5], there have been many analytic and numerical studies of $W_{n}$ in different random percolation problems 21]; it is of interest to extend such studies to the BCPM of the Ising model. On the other hand, we can extend the study $f_{n}(c)$ and $\langle c\rangle_{n}$ to the random percolation problem. It is interesting to compare the results for the BCPM and those for the random percolation problem. We may also extend the present study to the bond-diluted or the site-diluted Ising model which can be mapped into percolation models [22]. The criti- 
cal phenomena of the percolating properties of the Ising model are governed by the Ising fixed point (for example, $\nu=1$ ), whereas at the percolation threshold the critical phenomena are governed by the random percolation fixed point $(\nu=4 / 3)$. The crossover from the Ising fixed point to the random percolation fixed point in the process of dilution is highly interesting, especially for the properties depending on the number of the percolating clusters. The studies in these directions are in progress.
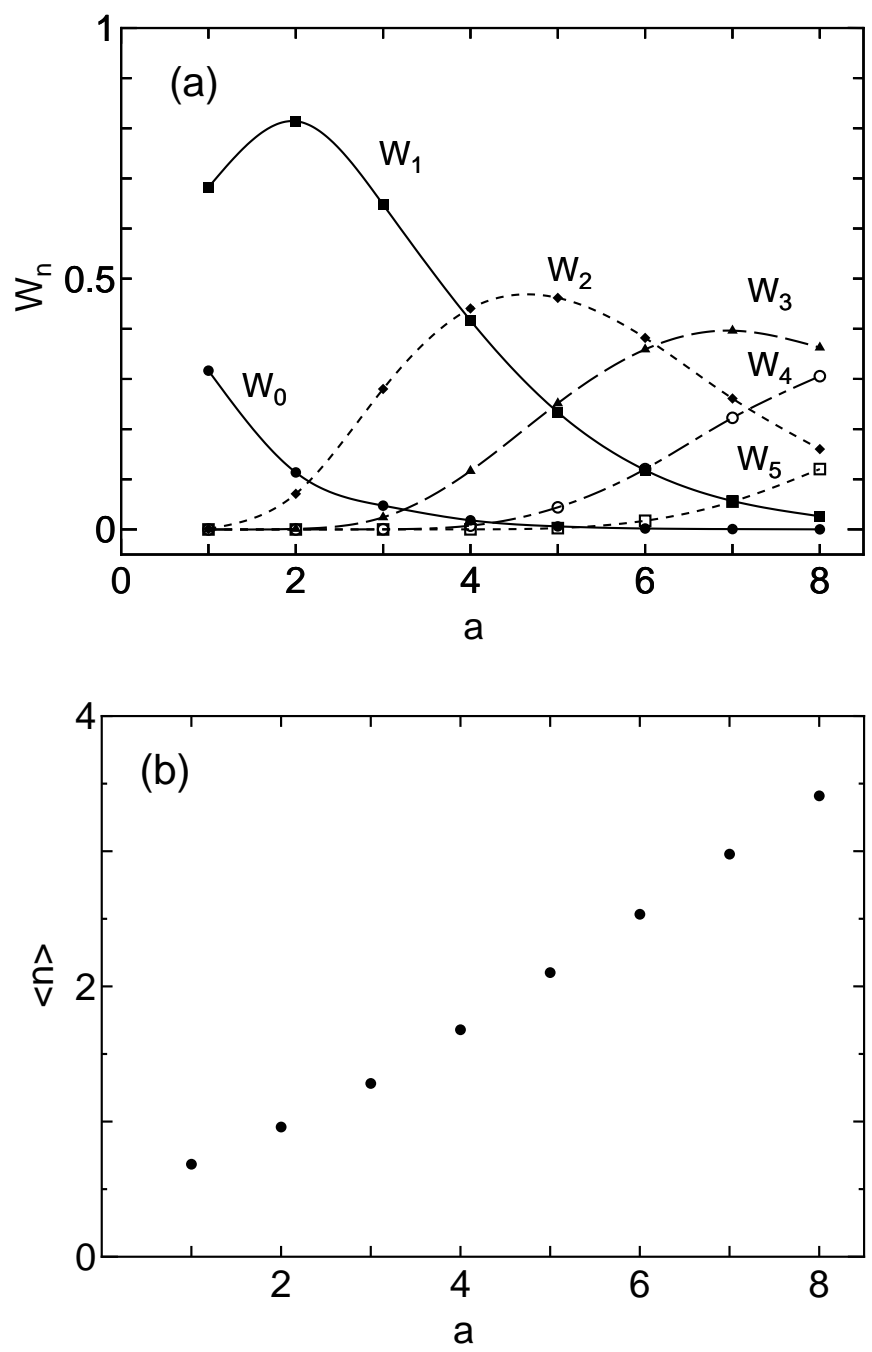

FIG. 4. (a) $W_{n}$ at $T=T_{c}$ as a function of $a=L_{1} / L_{2}$. (b) $\langle n\rangle$ at $T=T_{c}$ as a function of $a$.

We would like to thank M. Kikuchi and K. Kaneda for valuable discussions and the Supercomputer Center of the ISSP, University of Tokyo, for providing the computing facilities. This work was supported by a Grant-in-Aid for Scientific Research from the Ministry of Education, Science, Sports and Culture, Japan and by the National Science Council of the Republic of China (Taiwan) under grant numbers NSC 88-2112-M-001-011.
* $\quad$ Electronic address: okabe@phys.metro-u.ac.jp

+ Electronic address: huck@phys.sinica.edu.tw

[1] H. E. Stanley, Introduction to Phase Transitions and Critical Phenomena, (Oxford Univ. Press, New York, 1971).

[2] M. E. Fisher, in Proc. 1970 E. Fermi Int. School of Physics, edited by M. S. Green (Academic, New York, 1971) Vol. 51, p. 1; Finite-size Scaling, edited by J. L. Cardy (North-Holland, New York, 1988).

[3] V. Privman and M. E. Fisher, Phys. Rev. B 30, 322 (1984).

[4] D. Stauffer and A. Aharony, Introduction to Percolation Theory, Revised 2nd ed. (Taylor and Francis, London, 1994).

[5] C.-K. Hu, C.-Y. Lin, and J.-A. Chen, Phys. Rev. Lett. 75, 193 and 2786E (1995) and Physica A 221, 80 (1995); C.-K. Hu and C.-Y. Lin, Phys. Rev. Lett. 77, 8 (1996).

[6] Y. Okabe and M. Kikuchi, Int. J. Mod. Phys. C 7, 287 (1996)

[7] F.-G. Wang and C.-K. Hu, Phys. Rev. E, 56, 2310 (1997).

[8] C.-Y. Lin, C.-K. Hu, and J.-A. Chen, J. Phys. A 31, L111 (1998); C.-Y. Lin and C.-K. Hu, Phys. Rev. E, 58, 1521 (1998).

[9] C.-K. Hu, in Statistical Physics: Experiments, Theories, and Computer Simulations, edited by M. Tokuyama and I. Oppenheim (World Scientific, Singapore, 1998), p. 83; C.-K. Hu, J.-A. Chen, and C.-Y. Lin, Physica A 266, 27 (1999).

[10] Y. Okabe, K. Kaneda, M. Kikuchi, and C.-K. Hu, Phys. Rev. E, 59, 1585 (1999).

[11] C.-K. Hu, Phys. Rev. B 46, 6592 (1992).

[12] R. P. Langlands, C. Pichet, Ph. Pouliot, and Y. SaintAubin, J. Stat. Phys. 67, 553 (1992).

[13] K. Binder, Z. Phys. B 43, 119 (1981).

[14] C.-K. Hu, Phys. Rev. B 29, 5103 and 5109 (1984); for a review, see C.-K. Hu, in Computer-Aided Statistical Physics, edited by C.-K. Hu (AIP, New York, 1992), p. 79.

[15] P. W. Kasteleyn and C. M. Fortuin, J. Phys. Soc. Jpn. Suppl. 26, 11 (1969); C. M. Fortuin and P. W. Kasteleyn, Physica 57, 536 (1972).

[16] C.-K. Hu, Physica A, 189, 60 (1992); C.-K. Hu and J.-A. Chen, Physica A 199, 198 (1993).

[17] U. Wolff, Phys. Rev. Lett. 60, 1461 (1988).

[18] The definition of the metric factor $D_{1}$ is different in Ref. [6], where $t$ is defined by $T-T_{c}$.

[19] G. Kamieniarz and H.W. Blöte, J. Phys. A 26, 201 (1993).

[20] In [- [5], Hu and Lin used $R$ and $C(R)$ to represent $a$ and $\langle n\rangle$, respectively.

[21] M. Aizenman, Nucl. Phys. B 485 [FS], 551 (1997); J.-P. Hovi and A. Aharony, Phys. Rev. E 56, 172 (1997); J. Cardy, J. Phys. A 31, L105 (1998).

[22] C.-K. Hu, Phys. Rev. B 44, 170 (1991). 